\title{
In Vitro Diagnosis of Hypersensitivity to Nonsteroidal Anti-Inflammatory Drugs (NSAID) Comparison of Two Methods
}

\author{
Baló-Banga $\mathrm{JM}^{1^{*}}$ and Schweitzer $\mathrm{K}^{2}$ \\ ${ }^{1}$ Department of Dermatology, Medical Center of Hungarian Defense Forces, Budapest, Hungary \\ ${ }^{2}$ Department of Pathophysiology, Medical Center of Hungarian Defense Forces, Budapest, Hungary
}

"Corresponding author: Baló-Banga JM, Department of Dermatology, Medical Center of Hungarian Defense Forces, H-1062 Budapest, Podmaniczky u. 109-111, Hungary, Tel: +36-1-475-2628; Fax: +36-1-302-0347; E-mail: balmat05@freemail.hu

Received date: April 12, 2017; Accepted date: May 05, 2017; Published date: May 13, 2017

Copyright: ( 2017 Banga BJM, et al. This is an open-access article distributed under the terms of the Creative Commons Attribution License, which permits unrestricted use, distribution, and reproduction in any medium, provided the original author and source are credited.

\begin{abstract}
Objective: Current concept distinguishes between cross intolerance (non-immune) and single or multiple hypersensitivity based (immune) adverse reactions of non-steroidal anti-inflammatory drugs (NSAID) due to their potential to inhibit cyclooxygenase (COX) isoenzymes (COX-1, COX-2). Recently we described a rapid IL-6 release assay using blood mononuclear cells of patients with various clinical forms of drug hypersensitivity. Here we present data of a comprehensive analysis of the IL-6 release test and the classical IgE immuno-assay for their sensitivity in cases with adverse reactions to NSAIDs grouped according to the new clinical classification.
\end{abstract}

Methods: Total and specific serum IgE against 9 different HSA coupled-NSAIDs were determined by manual ELISA tests (55 cases) and compared to drug-specific release from preformed IL-6 pool of PBMCs of patients sensitized to the same NSAIDs after short (20) incubation of 4 standardized concentrations (51 cases and 9 controls) and IL-6 measurement from their cell free supernatants including positive and negative intraassay controls.

Results: The ratio of cross intolerant to specific hypersensitive (HS) cases was higher in the lgE group (and total $\lg \mathrm{E}$ too,) than in the IL-6 release tested ones. There was no difference, however, in the overall ratio of early and accelerated plus late onset adverse events based on individual histories. Nine NSAIDs were tested in both groups which represented all major COX-1 inhibitors. The positivity of validated test results was double within the IL-6 tested group $(65.4 \%$ vs. $36.9 \%)$. In some cases non-drug components of NSAID formulations were responsible for the observed (mainly) anaphylactic reactions. Positive results in both groups were scattered amongst cross intolerant and single to multiple hypersensitive (HS) subgroups. To our knowledge no comprehensive analysis had been performed before either on clinical phenotypes dependent IgE immunoassays or on NSAID-induced "early" Tcell activation after those specified adverse events.

Conclusion: Specific HS and multiple non cross-reactive NSAID sensitizations exceeded non- immune reactions in both in vitro tested groups. Some intolerant patients revealed detectable ASA antibodies of IgE type. Preformed IL-6 release by PBMC was more sensitive than specific IgE immunoassays as an in vitro diagnostic tool. The results indicate that checking of non-drug components should be considered in allergy workups. ASA in vivo provocations need further standardization.

Keywords: NSAID cross intolerance; NSAID hypersensitivity; COX-1; COX-2; Drug-specific serum IgE; IL-6 release; Provocation tests; Tablet additives

Abbreviations: Acet: Paracetamol; AGEP: Acute Generalized Erythematous Pustulosis; ANO: Angio Neurotic Oedema; ASA: Acetyl Salicylic Acid (Aspirin); bid: Twice A Day; CIU: Chronic Idiopathic Urticaria; Con A: Concanavalin A; Df: Diclofenac; DRESS: Drug Rash with Eosinophilia and Systemic Symptoms; HS: Hypersensitivity; HSA: Human Serum Albumin; IBD: Inflammatory Bowel Disease; Ibu: Ibuprofen; Indo: Indomethacin; LTT: Lymphocyte Transformation Test; Melox: Meloxicam; MEM: Minimal Essential Medium; MPE: Maculo-papular exanthema; Met: Metamisol; Nap: Naproxen; NECD: NSAID Exacerbated Cutaneous Disease; NERD: NSAID-Exacerbated Respiratory Diseases; NIUA: NSAID-Induced Urticaria/Angioedema; NSAID- Nonsteroid Anti Inflamatory Drugs; O.D.: Optical Density; PBMC: Perihperal Blood Mononuclear Cell; PBS: Phosphate Buffered
Saline; PHA: Phytohaemagglutinin-P; SDS: Sodium Dodecyl Sulfate; SDRIFE: Symmetrical Drug-Related Intertriginous and Flexural Exanthema; sNIUA: Single NSAID- Induced Urticaria/Angioedema; SNIUAA: Single NSAID-Induced Urticaria/Angioedema or Anaphylaxis; SNIDr: Single NSAID-Induced Delayed Reactions; TEN: Toxic Epidermal Necrolysis; UA: Urticaria Angioedema.

\section{Introduction}

Various NSAID inhibit cyclooxygenase isoenzymes (COX)-1 and -2 in plasma membranes to different extents. Because of their antipyretic and pain killing effect they are the mostly used drugs worldwide. Except for gastrointestinal and cardiovascular side effects they are responsible for the majority of drug hypersensitivity reactions [1] of both immunological and non-immunological types. Some years ago a unified classification by an expert panel has been published [2] in which there are two main groups: the "cross intolerance reactions" 
without allergic sensitization and the "classic drug allergic" ones. The former cause provocation by chemically non-related compounds, exclusively due to their relative affinity to COX-1 and COX-2 receptors in sensitive subjects. These can trigger either respiratory reactions like aspirin induced rhinosinusitis and asthma [3] or provoke cutaneous reactions like urticaria-angioedema (UA) progressing even to anaphylaxis. Chronic idiopathic urticaria (CIU) worsened by salicylates is a typical example and occurs in about $24 \%$ in hives patients [4]. Recently, basophil activation test and not serum IgE was proposed to indicate in vitro diagnosis in this subgroup of patients [5].

\begin{tabular}{|l|l|}
\hline NERD & NSAIDs ${ }^{*}$ - Exacerbated respiratory disease \\
\hline NECD & NSAIDs- Exacerbated cutaneous disease \\
\hline NIUA & NSAIDs ${ }^{*}$ - Induced urticaria/angioedema \\
\hline SNIUA & Single NSAID**-Induced urticaria/angioedema \\
\hline SNIUAA & Anaphylaxis \\
\hline SNDRs & Single NSAID ${ }^{* *}$-Induced delayed reactions \\
\hline
\end{tabular}

${ }^{*}$ Cross intolerance reactions

${ }^{* *}$ Cross reactivity with in chemically related drugs.

Table 1: Groups of NSAID hypersensitivity reactions.

Based on" true hypersensitivity", a substantial group of patients revealed similar skin symptoms as well as other organ involvement elicited by only one NSAID, mostly of high COX-1/COX-2 inhibitory potential, which might cross react with chemically related other drugs. The recent classification is based on these criteria. The reaction timing and clinical phenotypes are also of utmost importance separating immediate, early and delayed type manifestations [6]. Non-immediate urticaria and/or angioneurotic edema (sNIUA) as well as its progression to anaphylaxis (sNIUAA) and late exanthems can all be triggered by sensitized T-cells (Table 1). It is generally agreed that from the group of true drug hypersensitivity phenotypes those appearing within the boxes of Table 2 are delayed reactions - i.e. sNIDRs. Tests are thus mandatory. Current concept $[2,6]$ prefers in vivo methods that expose the patients, like prick-tests for screening, intradermal tests [7], of both early and late-reading, and provocation tests [8]-the gold standard. There are serious limitations, however. One of them is history of anaphylaxis albeit no validated data comparing its severity grades with systemic adverse effects of skin testing have been reported. It was stated, that except for pyrazolones, none of other NSAID chemical classes would be recommended for routine skin testing due to lacking standardization [7]. We attempted to fill in this gap and proposed uniformly 10-3M test solutions for a wide variety of drugs, including the NSAIDs; pyrazolons (enolic acid derivatives) DF (acetic acid derivative) and Ibu (propionic acid derivative) [9]. Oral provocation tests for aspirin are validated and are recommended to be performed even if another NSAID is suspected as a culprit in order to confirm or exclude COX-1 dependent cross-reactivity (NECD or NIUA-Table 1). These diagnoses arise if positive challenges even with minor symptoms occur after intake of both ASA and another chemically unrelated NSAID substance. The incremental doses of ASA starting from $10 \mathrm{mg}$ up to $500 \mathrm{mg}$ (cumulative dose) within one day are given according to established protocol [8]. No detailed descriptions for other NSAIDs oral tests could be found in the literature. Positive skin or general symptoms may arise within 4 hours after the last intake [10]. Patch test with ASA in particular and
Salycylic acid are performed in frames of "early urticaria test series" including food additives with reading times different from those for contact allergy (i.e. 20'-70'-24 hrs). A large series of photo patch testing has revealed $9.2 \%$ positivity with NSAIDs [11].

Many blood tests have been described in the past 60 years to diagnose adverse drug reactions. ASA specific IgE could be detected in a (most likely) sNIUA case [12]. Recent publications: fail to confirm the applicability of drug specific IgE determination in the differential diagnosis of NSAID hypersensitivity and raise the possibility of metabolites as culprit substances [13]. In earlier studies our group has compared drug-specific IgE levels in serum, binding to drug-HSA discs using ${ }^{125} \mathrm{I}$ coupled anti IgE and found high specificity but only $18.2 \%$ sensitivity against single dose non-blinded oral challenges in a retrospective study. Except for ASA, pyrazolons were also tested along with 5 different antibiotics [14] in hypersensitive and control cases but no detailed data for the various single drugs were evaluated. Cellular tests are difficult to perform, take days and are expensive. Pichler and Nyfeler found $78 \%$ sensitivity and $85 \%$ specificity of lymphocyte transformation test ( LTT) in 100 and 102 patients and stated that "pseudo allergies" to NSAID (i.e. Cross intolerance) were responsible for false positive results [15]. Basophil activation tests resulted in conflicting outcomes [16].

\begin{tabular}{|c|c|}
\hline Generalized urticaria $\pm \mathrm{ANO}^{1}$ & Stevens-Johnson syndrome \\
\hline Systemic Anaphylaxis \pm ANO & Purpurae +/-thrombopenia \\
\hline \multirow{3}{*}{$\begin{array}{l}\text { Generalized } \text { MPE }^{2}(<40 \%) \\
\text { Generalized MPE }(>40 \%) \\
\text { Fixed disseminated/bullous drug } \\
\text { eruption }\end{array}$} & Asthma, severe itch + ANO \\
\hline & Ulcerous mucosal manifestations \\
\hline & Generalized Pruritus \\
\hline Erythema multiforme & \multirow{4}{*}{$\begin{array}{l}\text { Erythema annulare Centrifugum /E. } \\
\text { nodosum } \\
\text { Flexural rash (SDRIFE }{ }^{3} \text { ) } \\
\text { AGEP }^{4} \\
\text { DRESS }^{5}\end{array}$} \\
\hline Toxic epidermal necrolysis- TEN & \\
\hline Small Patchy urticaria & \\
\hline Localized ANO & \\
\hline \multicolumn{2}{|l|}{ Circumscribed vesicular rash } \\
\hline \multicolumn{2}{|c|}{$\begin{array}{l}\text { 1. Angioneurotic edema; 2. Maculo-papular exanthema; 3. Symmetrical Drug- } \\
\text { Related Intertriginous and Flexural Exanthema; 4. Acute Generalized } \\
\text { Erythematous Pustulosis; 5. Drug Rash with Eosinophilia and Systemic } \\
\text { Symptoms; Strong boxes indicate typically "accelerated or late" occurring clinical } \\
\text { phenotypes fitting into sNIUA and sNI-Dr reactions. }\end{array}$} \\
\hline
\end{tabular}

Table 2: Phenotypic expression of drug hypersensitivity symptoms.

In our previous recent study we found that preformed IL- 6 released within 20 minutes at any or more of 4 different micro molar standard drug dilutions, tested on mononuclear cells above $50 \%$ over their diluents' levels, significantly correlated with the patient's history of drug-induced skin symptoms and with in vivo tests. Sensitivity of $85.4 \%$ and specificity of $82.4 \%$ of the IL- 6 release assay was found [1]. Out of 98 patients 58 had a positive history of one or more NSAID. Thirty per cent of all tests in the patients and $27 \%$ in the control group had been performed with these drugs [1].

Our aim was to compare 2 independent in vitro diagnostic tests; drug specific IgE ELISA determination from sera and the $20 \mathrm{~min}$. "early" T-cell activation detected by IL- 6 release by standard $\mu$ molar concentrations of various NSAIDs based on self-reported history or the symptoms seen. Cross-intolerance phenotypes were scrutinized for 
outcomes of in vitro test methods. In cases where in vivo test results were available, attempts were made to correlate them with in vitro results.

\section{Patients and Methods}

Retrospective analysis was performed among in- and out- patients of our teaching hospital between 2003 and 2013. The study was approved by the local Ethical Committee and patients gave their written consent to participate. Their symptoms were either seen by our staff or reported by the patients. The time elapsing between symptoms and tests was less than one year.

\section{In vitro Methods}

Two groups were formed with some overlapping cases. Group A consisted of 56 patients ( 36 women, mean age 46.6 yrs; 20 men, mean age 59 yrs.). Their sera taken after the adverse event was tested for specific IgE against various human serum albumin coupled NSAIDs: ASA, Ibu, Acet, DF, Indo, Pyrazolones (Met) and Oxicams by Hycor (Great Britain) manufactured EIA (122 tests). The manual method was used. Threshold for positivity was 0.35 O.D. units. "True" positive results were accepted at $>0.7$ O.D. [Class 2] and $>3.5$ O.D. (Class $3=$ strongly positive). In 14 cases the total IgE was co-determined. In 13 cases in vivo tests have been carried out along with the in vitro tests.

Group B consisted of 51 patients (45 women, mean age 44.3 yrs; 6 men, mean age 50.3 yrs.). In addition 9 control subjects ( 5 men and 4 women) with proven tolerance were tested as well. Their mean age was 50.3 years.

Isolation of PBMC on Ficoll-Paque (Amersham Bioscience UK) gradients followed by two washes with $\mathrm{PBS}$ and resuspension in Dulbecco's MEM containing $10 \mathrm{mM} \mathrm{CaCl}_{2}$ and $\mathrm{MgCl}_{2}$ each and $7 \mathrm{mM}$ of glucose (energy source) was performed as described earlier [1]. The incubation of $1.1 \times 10^{6} / \mathrm{ml}$ of PBMC ( $\geq 85 \%$ lymphocytes, viability $>95 \%$ ) without any plasma or serum was carried out in $450 \mu \mathrm{l}$ aliquots with PHA-P $(168 \mu \mathrm{g} / \mathrm{ml})$ or ConA $(5 \mu \mathrm{g} / \mathrm{ml})$ as positive and PBS as negative controls and 4 standard ( $\mu$ molar) dilutions $(1.5 ; 2.5 ; 3.5 ; 5.0)$ for each NSAID added in $50 \mu \mathrm{l}$ volumes. The final concentration for Asa, Ibu, Df, Acet, Met, Oxicams and additives were thus 10 times less (Figure 3). The incubation at $37^{\circ} \mathrm{C}$ for $20 \mathrm{~min}$. was finished by cooling and centrifugation at $30-50 \times \mathrm{g}$ for 6 minutes. Altogether 91 series $=546$ single tests were performed.

In some cases tablet additives: sodium lauryl sulfate (SDS) and ferric oxides (yellow, red, brown; molecular mass: 159.7 Madaus ${ }^{\mathrm{m}}$, Germany) were tested as well. After incubation cell free supernatants obtained were frozen at $-70^{\circ} \mathrm{C}$. In the second step released IL- 6 was measured by ELISA method using enzyme labeled monoclonal antibodies by Diagnosticum Ltd. Hungary [1]. Threshold for positivity was $+50 \%$ IL- 6 increases above negative (PBS) level at any of the 4 standard test concentrations provided that PHA-P or ConA samples were reactive as well. In 16 cases the total IgE was measured and in 18 cases in vivo tests have been performed within this group.

\section{In vivo Methods}

Patch tests have been carried out with ASA, Ibu, DF and Melox using $5-10 \%(\mathrm{w} / \mathrm{w})$ of pure substances dissolved in petrolatum. Readings at 20-40; 70' and 24 hrs for ASA +Salicylic acid (Brial Co, Germany) and 20-40', 48-72 hrs for other drugs, tested. Positive readings included contact urticaria and/or dermatitis $(1+-4+$ local strength). Intradermal tests have been performed using $10^{-3} \mathrm{M}$ solutions of pure substances in sterile saline compared to histamine $\left(10^{-4} \mathrm{M}\right)$ positive and (saline) negative controls. Threshold positivity was noted if urtica $>3 \mathrm{~mm}$ of diameter (d) and/or erythema $>25 \mathrm{~mm}^{2}$ developed within $20-40$ ' or papule $>3 \mathrm{~mm}$ (d) has occurred at $24 \mathrm{hrs}$ [9]. Oral challenges have been performed using single blind administration of a fraction of a tablet $(1 / 4$ or $1 / 2)$ given between 8-9 am. followed by close observation for $4 \mathrm{hrs}$ and telephone contact for additional $24 \mathrm{hrs}$. Positivity was accepted if skin or respiratory symptoms and/or $>20 \%$ deviation in vital parameters (blood pressure, pulse rate) have developed.

\section{Statistical Analysis}

\section{Summary statistics and two tailed t-tests have been used}

\section{Results}

1. Patients were (re) grouped according to the new classification as shown in Table 1 . Both test cohorts revealed the majority of cases within the single NSAID induced sNIUA as well as in sNIUAA fractions and less in the delayed type reactions (sNIDr). For this reason the two test series can be compared. In the present study Group $A=67 \%$; Group $B=73 \%$ of immediate-early reactions could be noted. Regarding overall distribution of tested NSAIDs within the two groups a similar pattern can be recognized with some differences; There were $33 \%$ more tests with ASA in the IgE than in the IL- 6 group, whereas $43 \%$ less tests of Df in the spec. IgE cohort as compared to the IL-6 group. The testing frequency was tailored according to each patient's individual history (Figure 1).

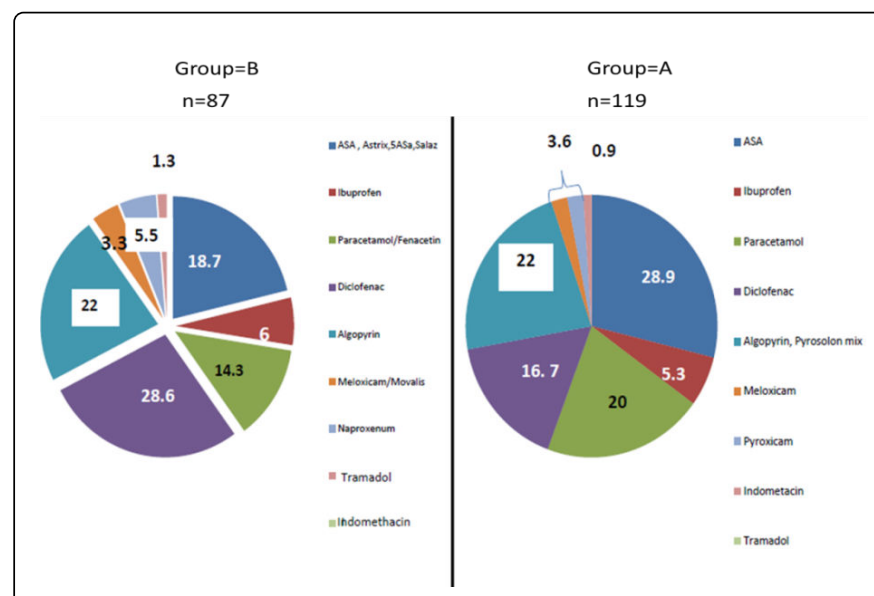

Figure 1: Distribution of tests due to various drugs within the two groups " $n$ " means the total tests in each group; in Group B only active cases (no controls) have been evaluated. Algopyrin=Metamisol (met).

The categories of "cross intolerance", respiratory symptoms dominance (NERD) and multivalent NSAID induced NIUA appeared mostly in the specific IgE group ( 9 and 7 cases). In NERD only ASA was positive; in 4/9 Class 2-3 and in 1/9 borderline positive cases. Two cases in this subgroup had suffered from typical symptom triad of Samter [15]. In suspected NIUA (NECD) cases of the specific IgE group only one reacted positively (Class 2 units) to ASA and DF. The remaining 6 revealed 13 negative or weakly positive (Class 1 ) results to 
various drugs; ASA, Df, Ibu, Met, Acet. The clinical phenotype in all cases was urticaria and ANO. Three positive provocation tests occurred. The sNIUA cases of Group A revealed 14/30 (47\%) positive specific IgE results with highest prevalence of Melox (1/1), Ibu (2/3) ASA (5/9), followed by Met (4/8), Acet and Df (both 1/3). NERD was suspected in the IL-6 release group in one case. ASA was not tested but Df was weakly positive. In the NIUA suspect fraction of Group B, 3 cases were included. All had urticaria and ANO and multiple NSAID induced reactions with preferential localization in the perorbital area. All of 7 tests with various drugs were positive. Among them one patient (23 years man) has suffered from polyvalent allergies including various foods, pollens and house dust mite. Within the sNIUA cases of Group B there were 24/32 (75\%) positive IL-6 release results. The highest prevalence occurred with Nap (2/2), followed by Met $(8 / 10)$, ASA (5/7), and Df (4/8), Melox (1/2), Ibu (1/2). It has to be mentioned that one of 2 tests with Ibu was false negative, proven by intradermal test positivity later. The least positive results $(1 / 4)$ were encountered by testing Acet and Tramoxenic acid (0/1). The sNIDr fraction of Group A contained 18 patients and 9 were positive at test rates 13/34 (38\%). There were equally $4 / 13$ positive tests for ASA and Met; $3 / 13$ for Acet and $2 / 13$ for Df. Ibu was negatively tested. Within the sNIDr fraction of Group B 13 patients and 19 tests were considered. Out of them 10 had positive results, one of whom had tolerated the drug tested. Thirteen tests were positive and 7/13 (54\%) single drug positivities had occurred, while 3 patients showed reactions to 2 unrelated drugs. Three patients have revealed 6 (only) negative reactions. Df, Acet and Met were positively tested at equal higher rates while ASA and Ibu at equal low rates. The most frequent diagnoses within the sNIDr groups were maculo-papular rashes, fixed drug exanthems, vasculitis and purpurae, DRESS, late onset urticaria, prurigo and SDRIFE*

2. Total IgE levels: In Group A significantly elevated total IgE (363 $\mathrm{kU} / \mathrm{l} \pm 83$ s.e.m.) over Group B $(89.7 \mathrm{kU} / \mathrm{l} \pm 28.3$ s.e.m. $)$ was noted. Two-tailed probability gave $\mathrm{p}=0.0067$ value. In spite of the significant difference, both groups revealed many positive specific IgE results against pollens, food antigens and to other drugs, mostly to antibiotics.

3. Comparison of humoral (spec IgE) and cellular (PBMC IL-6 release) tests (Table 3.): Criteria upon which the 2 tests could be compared regarding negativity and positivity taking into account the grading as well are demonstrated in Table 3 . There were nearly the same rates of negative results in the two groups. The corresponding definitions are explained in column 1 for the specific $\operatorname{IgE}$ determinations and in column 4 for the IL- 6 release assay.

Positive test rates are also similar except for strong positivity occurring in IL-6 tested cases with more than double frequency. There were 8 cases (all women) and 9 tests in this group.

\begin{tabular}{|l|l|l|l|l|l|}
\hline Definition 1 & Result 1 & Spec. IgE (\%)n=119 & Definition 2 & Result 2 & $\begin{array}{l}\text { IL-6 release } \\
\mathbf{( \% )} \mathbf{n}=\mathbf{8 7}\end{array}$ \\
\hline$<0,35$ O. D. & Negative & 34.4 & No signif. release & Negative & 34.3 \\
\hline Class 1 & weakly + uncertain & 28.6 & single peak, except at $0.15 \mu \mathrm{M}$ & weakly + & 16.1 \\
\hline Class 2 & positive & 29.4 & $>1$ peaks or one at $0.15 \mu \mathrm{M}$ & positive & 35.8 \\
\hline Class 3 & strong pos. & 5.0 & $>1$ peaks incl. one at $0.15 \mu \mathrm{M}$ & strong pos. & 12.7 \\
\hline$\sim 0,35$ O.D. & undefined, negative & 2.5 & Any release, not $>(+) 50 \%$ backgr. & Undefined negative & 0 \\
\hline
\end{tabular}

Definition 1 is the widely accepted class grading (25) except for the fluorescent enzyme immunoassay (FEIA-ref 29); Definition 2 was created from the previous work of our group (ref 1) Left side columns /Group A/, right side columns /Group B/. Column 6 reflects the results without the 9 control person's 16 negative tests (validated by in vivo tolerance) because in Group A there were no negative controls included.

Table 3: Evaluation of relative frequencies of negative and positive results obtained by the 2 tests.

Upon comparison with Group A in Group B 4 of eight cases with strong positivity belonged to late reactors (sNIDr), 3 cases to sNIUA and one to sNIUA-Anaphylaxis (Table 1). Seven of 9 tests with various NSAIDs and 2/9 with SDS gave strong positive results. The total IgE values measured in this group fell into negative range $(20-30 \mathrm{kU} / \mathrm{l})$. The four strong reactors ( 5 tests) of Group A were women, 3 of them belonged to sNIUA and one to sNIDr. All had very high specific IgE values.

4. The negative, weakly positive and undefined results amounted altogether to $65,5 \%$ of specific IgE tests (lines $2+3+6$, column 3 ). Within the Group B even slightly positive results (IL- $6>+50 \%$ at any of specified drug dilutions-see definition) could be clinically validated as true ones. This has resulted in $65.5 \%$ overall positivity rate of this test (lines $3+4+5$, column 6 ) against $34.5 \%$ of group A. While ASA was tested most frequently in Group A resulting in $55.2 \%$ positivity (Classes 2-3) all other drugs yielded only from 25\% (Df, Oxicams) to $33.3 \%$ (Ibu) "true" positive results in all subgroups together. Acet and Met were at 30.4 and $30.3 \%$, respectively.

${ }^{*}$ listed but not given in weight
In the IL- 6 release assay the most frequently tested drug was Df with $41 \%$ positive results, followed by Met and ASA revealing $83.3 \%$ and $76.5 \%$ positivity. Nap (Propionic acid derivative) was positive in all tested cases. Melox tests were equally positive and negative (50\%).

5. Non-NSAID ingredients of tablets as culprit substances could be detected by the IL- 6 release assay. In some unexpectedly occurring negative results, other non-drug components of the formulation e.g. SDS in some ASA tablets or ferric oxides in Df tablets were tested positively. Figure 3 demonstrates the results obtained by IL- 6 release from sensitized mononuclear cells due to the suspected culprit drug ASA and tablet ingredient SDS. The results of two independent experiments (and times) are shown. While rapid onset purpura on the legs persisted, the $64 \mathrm{y}$ old woman revealed negative IL- 6 test to aspirin which she took while the rash has appeared. When the drug was stopped symptoms have gradually disappeared. Four months later we decided to test SDS (unknown ${ }^{*}$ amount in the stomach protective ASA tablet). Strongly positive result has occurred. In vivo proof has been obtained later as the patch test was highly positive as well. The 
Citation: Baló-Banga JM, Schweitzer K (2017) In Vitro Diagnosis of Hypersensitivity to Nonsteroidal Anti-Inflammatory Drugs (NSAID) Comparison of Two Methods. J Allergy Ther 8: 256. doi:10.4172/2155-6121.1000255

Page 5 of 8

patient continued to take another ASA formulation without SDS and has remained symptom free. Ferric oxides as tablet colorants have the same molecular weight but their different color depends on shape and size of particles. All four patients tested were women and the suspected drug was Df. Three patients had negative in vitro results and only one was positive with Df. Two of the 4 showed anaphylaxis and emergency unit care was necessary. The other 2 had suffered from widespread UA rash. "Yellow" $\mathrm{Fe}_{2} \mathrm{O}_{3}$ was positive in all of them as well as in one of 4 tests the "brown" iron oxide stain. Two patients were tested with the red colorant as well but were negative.

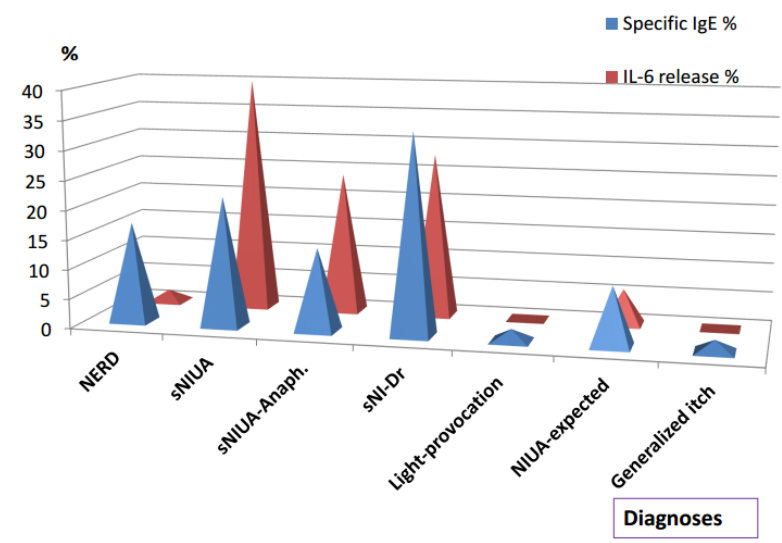

Figure 2: Percentual distribution of patients tested by different methods due to internationally agreed phenotypic classification.

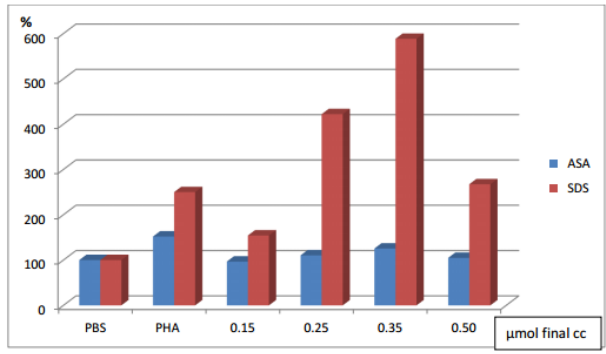

Figure 3: The effect of allergy eliciting hapten on the release of IL-6 from sensitized PBMCs. SDS is an additive within some ASA tablets. Test with ASA: negative, patient has tolerated ASA tablets without SDS. Test with SDS positive!.

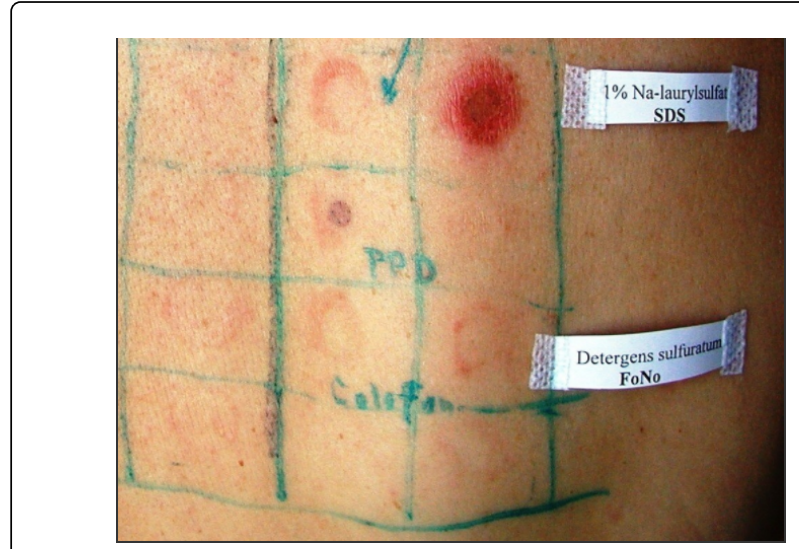

Figure 4: Patch test performed to prove SDS hypersensitivity in a 64 year old women who took gastric mucosa protective ASA tablets (reading after $48 \mathrm{hrs}$ ). Her in vitro results are shown in Figure 3. Arrow indicates methyl-isothiazolidin $(0.1 \%)$; PPD=paraphenylene diamine free base ; Colofon=colofony; Detergens sulfuratum FoNo (Formulae Normales)=medicinal hair shampoo in Hungary, containing small amount of SDS.

6. In vivo - in vitro comparisons: In 16 patients of the IgE test group seven skin tests (intradermal, patch) and 9 provocations have been performed. Out of 11 positive reactions, one patch, 2 intradermal and 8 provocations were observed. The most positive results were obtained with ASA (7) followed by Df (2) and Ibu (2). In patients of the IL-6 group 21 in vivo parallel test were carried out. Out of 8 positive reactions one patch and 7 provocations have been performed. ASA, Df and Ibu were positive in 2 cases (each) Acet and Met in one case (each). In addition, 13 tests in 8 tolerant control subjects of this group were negative. In one control case however, $125 \mathrm{mg}$ of oral Met intake was tolerated while $250 \mathrm{mg}$ caused mild symptoms within the observation period. Figure 5 shows positive patch tests with two chemically different NSAIDs. Figure 6 demonstrates disseminated red spots on the neck due to positive patch testing with ASA (20-70 on the back and subsequent spreading.

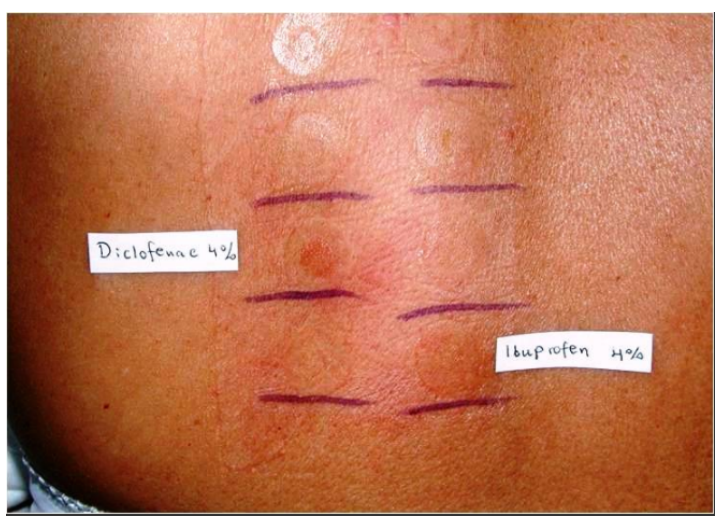

Figure 5: Multiple positive patch tests (reading after $48 \mathrm{hrs)} \mathrm{by} \mathrm{Df}$ (acetic acid derivative) and Ibu (arylpropionic acid derivative) after non-immediate but accelerated (sNIUA) symptoms in a $64 \mathrm{y}$ old woman. 


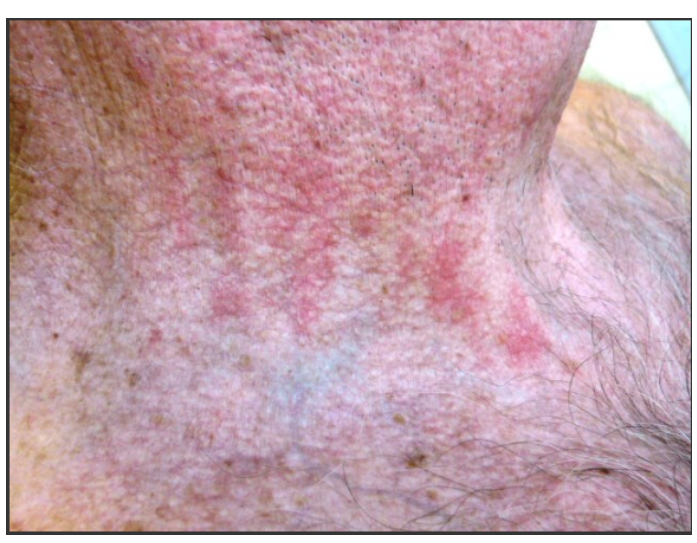

Figure 6: Flare up of small patchy urticaria on the neck of a $72 \mathrm{y}$ old man 70 after the start of patch tests with 5\% ASA and 10\% Salicylic acid. The patient had mild but persistent symptoms to Pentasa (methylsalicylate) taken because of IBD.

\section{Discussion}

In an earlier series of our group the ratio of immediate-early to delayed reactions was 6:4 [10]. Since that time a new clinical classification has emerged (Table 1). Our results pointed to the fact that hypersensitivity related phenotypes (sNIUA, sNIUAA, sNIDr-see Table 1) to various NSAIDs clearly exceeded those of presumed "cross intolerance" cases without true sensitization. The higher representation of NECD (9/56) and of NIUA (7/56) in Group A against those (1/51) and (3/51), respectively, in Group B reflected the first line selection of IgE-based diagnostics for rapid onset respiratory and urticarial reactions. This is also expressed by significant difference in total $\operatorname{IgE}$ values. The specific IgE tests showed within the NERD subgroup 5/9 negative results and $4 / 9$ true ( $>=$ Class 2 ) positive readings with ASA. Out of them one of two with Samter triad [16] and IgE positivity has tolerated even $200 \mathrm{mg}$ of aspirin given in gradually increasing amounts under ward conditions. The other patient with negative IgE result to ASA has developed respiratory symptoms to $100 \mathrm{mg}$ ASA given orally without known previous sensitization but turned to tolerate this drug if $50 \mathrm{mg}$ bid was administered. Both decided to continue ASA treatment because of marked clinical improvement of asthma and rhinoconjunctivitis. Thus, individual follow-up would be needed as stressed [17]. In the latter only patient out of 9 we obtained one "true" specific IgE positivity with Acet and 2 other questionable results (Class 1 each) with Df and Met. All test results with other NSAIDs in this group were negative. Our data indicated that neither fractionated provocations nor specific IgE present in some of the patients' sera were useful as adjunct diagnosis of NERD. The only patient with suffocation after various drugs and suspected NERD within Group B was weakly positive to Df and oral provocation to Met was negative. Due to lacking history no test with ASA has been initiated. In a recent publication the heterogeneity of NERD group is stressed and oral, bronchial or nasal challenge only with ASA is recommended as an ultimate tool of diagnosis. Authors denied both skin testing and in vitro methods with culprit drugs [3]. In our opinion this oversimplification does not allow either a better insight into mechanisms in play nor would comply with differential diagnostic approaches. The link between NIUA and atopic disease has been raised earlier. A certain NSAID cross-reactive subset of patients could develop periorbital ANO and reveal house dust mite sensitization, i.e. extrinsic type of atopic disease with increased $\operatorname{IgE}$ [18]. This symptoms-association was reported in children and adults as well and termed "isolated periorbital oedema"/IPO/and distinguished because of lack of CIU but a separate clinical entity was not accepted for these patients by opinion leaders [19]. Our results have shown that, in contrary to NERD, in NIUA cases the use of specific IgE as diagnostic tool could not be argued for. Most interestingly, IL-6 release tests showed multiple sensitization pattern [20] which differed from sNIUA cohort of Group A. In this subgroup B, $25-80 \%$ of tests have been positive to various degrees with various drugs. The highest positive rates provoked by Nap, Ibu and ASA correlated with high relative COX1/COX2 inhibitory rates for these drugs; (ASA $\sim 500$, Naproxen $\sim 5-6$ and Ibuprofen $\sim 3-4$ ) [21]. The multiple sensitization i.e. more than one positive test result with chemically related NSAIDs was $23 \%$ in Group A and $42 \%$ in Group B. due to higher overall positivity rates of the latter. According to Pichler and coworkers this can be explained by the $\mathrm{p}-\mathrm{i}$ (pharmacological interaction) concept as well. Therefore, these results are not related to the cross-intolerance phenomenon seen in NERD or NIUA [22] and can be regarded as "flare-ups" depending on the high level of T-cell reaction elicited by the first event but disappearance later with elapsing time. Moreover, recent work has stated multiple NSAID hypersensitivity without allergy to aspirin [23]. From our sNIUA cases of Group A 2/3 were "true" positive to ASA and from sNIUA of Group B 4/8 tested for ASA due to history-reacted all positively. IL-6 release assay results therefore, were of no help separating NIUA and sNIUA subgroups as distinct clinical entities. The higher incidence of negative tests by Df and Melox might reflect their lower potency of COX1/COX2 inhibition [3] well established by now for each NSAIDs in use [21]. The sNIDr phenotypes showed the most single drug induced positive tests in both Groups (A\&B). In Group A $4 / 18$ patients had multiple hypersensitivities with chemically non-related drugs. In Group B 2/13 patients reacted positively with chemically distinct drugs ( 2 each). The most frequent associations in both test cohorts were Met (Pyrasolonenolic acid derivative) and Acet (p-aminophenol derivative). The mechanism of "early" release of preformed IL-6 from mononuclear cells has been studied after stimulation by mitogens and by chemical allergens such as drugs and Nickel ions [1]. It might represent the earliest cellular phase of various allergic reactions. The key event is the presentation of the sensitizing molecule. IL- 6 is released almost immediately from its preformed pools and binds to both its soluble and cell membrane attached receptors. Furthermore, it also binds to the ubiquitous membrane receptor gp 130 and through this "transsignaling" and forming of a "functional receptor complex" an immune response could start. Except for "turning on" antigen presenting monocytes, lymphocyte activation starts as well and T-cell proliferation would occur [24]. We found that multiple test concentrations were needed to detect total sensitivity related to clinical phenotypes [1].

More severe or widespread rashes tended to release significant ( $>50 \%$ over background) IL- 6 at the lowest or at multiple drug concentrations. The grading of test positivity was based on these observations (Table 3 ) and supported also by our previous results on a large group of various drugs [1]. The serum specific IgE levels were divided into classes which indicate tenfold increases of analyte in each step (Classes 1-4). It is generally agreed that only class 2 or greater could be interpreted as clinically significant or positive. Below this, class 1 is dubious or negative and 0 is definitely negative [25]. This was the basis of our comparison as detailed in Table 3. The good correlation of the percentages of negative and of undefined negative 
results within the Groups A and B could argue for the comparability of the two groups. The approximately two fold increase of positivity rates in favour of IL-6 release tests stresses its applicability in the differential diagnosis of NSAID-induced adverse reactions. There is no need for sterile cell cultures and much less time is necessary to obtain results. The lack of sensitivity of available NSAID-specific IgE assays does not exclude the role of IgE-mediated clinical presentations or skin test results [26]. Recently, Steiner et al., listed a number of publications considering data for NSAID specific IgE tested in "early onset" hypersensitivity reactions as "not available" [27]. Single drug tests to the pyrazolone derivate propyphenazon were of high diagnostic value though [28].

Our results could at least partly fill in this gap, regarding other NSAIDs. It is important to mention that modern automated analyzers would be much less sensitive in detecting pathologically elevated NSAID-specific IgE levels than the "older" manual ELISA test systems [29]. The finding of HS related to non-drug components of some NSAID formulations was established by using the more sensitive IL-6 release test and this could widen our horizon looking into adverse reactions occurring after taking "pain-killers" or anti-thrombotic drugs. The weakness of the above studies is the lack of ASA tests for all patients in all cross-intolerant subgroups to match with the provocation tests. One patient in the sNIUA subgroup A who reacted positively to oral Nimesulid (COX-2 antagonist) had positive skin tests to various sulphonamides, a basic structure to all COX-2 antagonists in use.

The aim of future clinical research is to differentiate between cross intolerance reactions of selective COX-1 inhibitors and multiple allergy syndrome involving both COX-1 and COX-2 inhibitors.

\section{Conclusion}

T-cell IL-6 release measured after short incubation from supernatants can be recommended to supplement to or substitute for in vivo testing after hypersensitivity events to NSAIDs. Specific IgE determinations are of limited value because of high false negativity. The intolerance reactions with no HS need a different allergy workup starting with in vivo tests with ASA. The majority of our skin patients did not fall into these categories. Multiple non cross-reactive HS was common among early accelerated phenotypes.

\section{Funding Source}

This work was supported by the Hungarian Research Grants OMFB-00284/04 and OMFB- 00285/04.

\section{Conflicts of Interest}

Authors declare no conflict of interest.

\section{Acknowledgements}

Authors are indebted to L.A. Réthy, MD, K. Rásky and Prof. K. Balogh for critically reviewing the manuscript.

\section{References}

1. Baló-Banga JM, Schweitzer K, Lakatos S, Sipka S (2015) A novel rapid (20-minute) IL-6 release assay using blood mononuclear cells of patients with various clinical forms of drug induced skin injuries. World Allergy Organ J 8: 1-11.
2. Kowalski ML, Asero R, Bavbek S, Blanca M, Blanca-Lopez N, et al. (2013) Classification and practical approach to the diagnosis and management of hypersensitivity to nonsteroidal anti-inflammatory drugs. Allergy 68: 1219-1232.

3. Makowska J, Lewandowska-Polak A, Kowalski ML (2015) Hypersensitivity to Aspirin and other NSAIDs: Diagnostic Approach in Patients with Chronic Rhinosinusitis. Curr Allergy Asthma Rep 15: 47-58.

4. Doeglas HM (1975) Reactions to aspirin and food additives in patients with chronic urticaria, including the physical urticarias. Br J Dermatol 93: 135-144.

5. Hsieh CW, Lee JW, Liao EC, Tsai JJ (2014) A Disease Marker for AspirinInduced Chronic Urticaria. Int J Mol Sci 15: 12591-12603.

6. Demoly P, Adkinson NF, Brockow K, Castells M, Chiriac PA, et al. (2014) International consensus on drug allergy (Position paper). Allergy 69: 420-437.

7. Brockow K, Garvey LH, Aberer W, Atanaskovic-Markovic M, Barbaud A, et al. (2013) Skin test concentrations for systematically administered drugs-an ENDA/EAACI drug allergy Interest Group position paper. Allergy 68: 702-712.

8. Nizankowska-Mogilnicka E, Bochanek G, Mastalerz L, Swierczynska M, Picado C, et al. (2007) EAACI/Ga2Len guideline aspirin provocation tests for diagnosis of aspirin hypersensitivity. Allergy 62: 1111-1118.

9. Baló-Banga JM, Vajda (2014) Attempts to standardize intradermal drug tests based on molecular mass and on clinical phenotypes. Some pitfalls or exceptions?. Clin Transl Allergy 4: P102.

10. Réthy LA, Baló-Banga JM (2004) The allergic and other side effects of non-steroid anti-inflammatory drugs and gold salts. Orv Hetil 145: 1943-1949.

11. Przybilla B, Ring J, Schwab U, Galosi A, Dorn M, et al. (1987) Photosensitizing properties of nonsteroidal antirheumatic drugs in the photopatch test. Hautarzt 38: 18-25.

12. Blanca M, Perez E, Garcia JJ, Miranda A, Terrados S, et al. (1968) Angioedema and IgE antibodies to aspirin: a case report. Ann Allergy 62: 295-298.

13. Bolze S, Bromet N, Gay-Feutry C, Massiere F, Boulieu, et al. (2002) Development of an in vitro screening model for the biosynthesis of acyl glucuronide metabolites and the assessments of their reactivity toward human serum albumin. Drug Metab Dispos 30: 404-441.

14. Baló-Banga JM, Németh G, Jacobsen HJ (1994) Comparative studies on drug-specific IgE, lymphocyte chromatin activation and oral challenge tests in drug allergic subjects. Magyar Belorvosi Archivum 5: 395-398.

15. Nyfeler B, Pichler WJ (1997) The lymphocyte transformation test for the diagnosis of drug allergy: sensitivity and specificity. Clin Exp Allergy 27:175-181.

16. Samter M, Beers RE (1968) Intolerance of aspirin. Clinical studies and consideration of its pathogenesis. Ann Intern Med 68: 975-983.

17. Gosepath J, Schaefer D, Amedee RG, Mann WJ (2001) Individual monitoring of aspirin desensitization. Arch Otolaryngol Head Neck Surg 127: 316-321.

18. Quiralte J, Ávila-Castellano R, Cimbollek S (2014) A phenotype-based classification of NSAIDs hypersensitivity: new apetients, new challenges. Allergy 69: 81.

19. Kowalski ML, Makowska J (2014) Reply: To PMID 24117484. Allergy 69: 815-816.

20. Macy E (2004) Multiple antibiotic allergy syndrome. Immunol Allergy Clin North Am 24: 533-543.

21. Brune K, Partignani P (2015) New insights into the use of currently available non-steroidal anti-inflammatory drugs. J Pain Res 8: 105-118.

22. Pichler WJ, Daubner B, Kawabata T (2011) Drug hypersensitivity: flareup reactions, cross- reactivity and multiple drug hypersensitivity. J Dermatol 38: 216-221.

23. Pérez-Sanches N, Bogas G, Cornejo-Garcia JA (2016) Multiple NSAID hypersensitivity without hypersensitivity to aspirin. J Allerg Clin Immunol Pract 4: 524-525. 
Citation: Baló-Banga JM, Schweitzer K (2017) In Vitro Diagnosis of Hypersensitivity to Nonsteroidal Anti-Inflammatory Drugs (NSAID) Comparison of Two Methods. J Allergy Ther 8: 256. doi:10.4172/2155-6121.1000255

Page 8 of 8

24. McLoughlin RM, Jenkins BJ, Grail D, Williams AS, Fielding CA, et al (2005) IL-6 trans-signaling via STAT3 directs T cell infiltration in acute inflammation. Proc Nat Acad Sci U S A 102: 9589-9594.

25. Sanz ML, Prieto I, García BE, Oehling A (1996) Diagnostic reliability considerations of specific IgE determination. J Investig Allergol Clin Immunol 6:152-161.

26. Stone SF, Phillips EJ, Wiese MD, Heddle RJ, Brown SG, et al. (2013) Immediate type hypersensitivity drug reactions. Brit J Clin Pharm 78 $1-13$.
27. Steiner M, Harrer A, Himly M (2016) Basophil reactivity as Biomarker in immediate drug hypersensitivity reactions. Potential and limitations. Front iers in Pharmacology 7: 171-183.

28. Himly M, Jahn-Scdhmid B, Pittertschatscher K, Bohle B, Grubmayr K, et al. (2003) IgE-mediated immediate type hypersensitivity to the pyrazolone drug propyphenazone. J Allergy Clin Immunol 111: 882-888.

29. Mayorga C, Sanz ML, Gamboa PM, Garcia BE (2010) In vitro diagnosis of immediate allergic reactions to drugs: An update. J Invest Allergol Clin Immunol 20: 103-109. 